\title{
Germanica
}

\section{Réflexions sur le lyrisme de Erich Kästner durant la phase finale de la «Neue Sachlichkeit»}

$\mathrm{Zu}$ Erich Kästners lyrischen Schaffen in der Endphase der Neuen Sachlichkeit

\section{Pierre Vaydat}

\section{(2) OpenEdition}

\section{Journals}

Édition électronique

URL : http://journals.openedition.org/germanica/2092

DOI : 10.4000/germanica.2092

ISSN : 2107-0784

Éditeur

Université de Lille

\section{Édition imprimée}

Date de publication : 1 janvier 1992

Pagination : 83-96

ISSN : 0984-2632

\section{Référence électronique}

Pierre Vaydat, « Réflexions sur le lyrisme de Erich Kästner durant la phase finale de la «Neue Sachlichkeit» », Germanica [En ligne], 10 | 1992, mis en ligne le 12 février 2014, consulté le 06 octobre 2020. URL : http://journals.openedition.org/germanica/2092 ; DOI : https://doi.org/10.4000/ germanica.2092

Ce document a été généré automatiquement le 6 octobre 2020.

(C) Tous droits réservés 


\title{
Réflexions sur le lyrisme de Erich Kästner durant la phase finale de la «Neue Sachlichkeit»
}

\author{
Zu Erich Kästners lyrischen Schaffen in der Endphase der Neuen Sachlichkeit
}

\author{
Pierre Vaydat
}

1 Les considérations qui suivent laissent délibérément de côté le reflet direct de la réalité contemporaine dans les poèmes du jeune Kästner. Cet aspect documentaire a fait l'objet de plusieurs études qui en ont abondamment dégagé l'intérêt ${ }^{1}$. Mon intention est de faire ressortir ce qui donne aux poèmes des quatre recueils Herz auf Taille, Lärm im Spiegel, Ein Mann gibt Auskunft et Gesang zwischen den Stühlen², publiés entre 1928 et 1932, leur tonalité personnelle.

2 De prime abord, le lyrisme n'a pas sa place dans le programme de la «Nouvelle Objectivité ", puisqu'il est aux antipodes du constat, du réalisme sociologique et des attitudes extraverties. L'esthétique du réalisme nouvelle manière affecte de mépriser la sentimentalité traditionnelle du moi lyrique; elle est fondée sur une philosophie qui congédie le transcendant et les relations magiques avec le cosmos, car elle récuse dans sa volonté de lucidité l'anthropomorphisation du monde. Or, sans le transfert plus ou moins spontané à Dieu et à la Nature des qualités humaines, l'univers poétique devient, semble-t-il, inconcevable. Il n'en demeure que des moules formels dont le mythe s'est retiré. Le lyrisme du jeune Brecht est bien dans la logique du projet néo-réaliste lorsqu'il utilise des strophes et des mètres qu'un usage séculaire a rendu vénérables pour les faire servir, dans une intention provocatrice, à une édification anti-chrétienne, anti-romantique, à un didactisme au service d'une subversion permanente de la société autoritaire. L'objectivité froide qu'affiche la «Neue Sachlichkeit» ne saurait admettre théoriquement qu'une poésie dont le but serait d'instruire et de critiquer : une poésie qui serait d'une part nettement didactique, qui tiendrait de la fable, de la parabole sécularisée, et qui serait surtout satirique, mais en restant dans le registre de la dérision, sans jamais se parer d'accents prophétiques laissant transparaitre une inspiration divine. Kästner marque bien sa fidélité à ce programme lorsqu'il professe de 
«fabriquer des vers» (cf. la «Versfabrik» du poème «Kurzgefasster Lebenslauf», strophe 5, dans Ein Mann gibt Auskunft); et lorsqu'il déclare produire un lyrisme pour « usagers » («Gebrauchslyrik»; cf. la «Prosaische Zwischenbemerkung» dans Lärm im Spiegel). Le terme «Gebrauch» vient d'ailleurs de Brecht, qui écrivait en 1927 dans sa préface à la «Hauspostille»: «Die Hauspostille ist für den Gebrauch der Leser bestimmt».

3 Il y a dans cette modestie une part d'affectation. Car il ressort clairement du texte Prosaische Zwischenbemerkung que le «Gebrauchspoet», loin d'être un simple fabricant ou un humble fournisseur, crée une poésie authentique, et qu'il est même plus proche intrinsèquement des grands lyriques du passé que ceux qui les imitent de façon servile, empruntant leurs thèmes, leurs images et leurs rythmes sans produire autre chose que des répliques factices et donc ennuyeuses, bien qu'elles témoignent parfois d'une habileté technique incontestable: "Günstigstenfalls klingen ihre Gedichte. Aber es steckt nichts drin. Was sollen sie andres machen: sie klingen hohl!». Faut-il en conclure pour autant que l'entreprise de dépoétisation menée par Kästner («Ich gehe durch den Garten der Gefühle/Die tot sind, und bepflanze sie mit Witzen», cf. Kurzgefasster Lebenslauf, strophe 6) est une pose, une attitude non dépourvue d'arrière-pensées publicitaires, plutôt qu'une profession de foi ? Kästner est bien entendu sincère, mais il faut tenir compte du fait qu'il est un humoriste, et qu'il lui arrive de se moquer de cette «Sachlichkeit» devenue un terme à la mode. Songeons par exemple à un titre comme Sachliche Romanze (dans «Lärm im Spiegel»). Faut-il, inversement, prendre intégralement au sérieux un programme qui est surtout une réaction excédée face aux pâmoisons et aux vaticinations d'un certain expressionnisme et qui, lorsqu'il est formulé dogmatiquement, comme par exemple dans le compte rendu d'Ernst Glaeser sur le roman d'Erik Reger ${ }^{3}$ Union der festen Handl, tourne lui aussi à la caricature? Kästner est un esprit trop fin et trop ironique, trop foncièrement négateur aussi, pour se laisser ligoter par des préceptes de théoricien. Il n'empêche que notre poète, si l'on considère l'ensemble de ses textes "weimariens», ne se laisse aller à l'effusion que très rarement, et que dans sa production le désenchantement l'emporte; non pas le désenchantement romantique d'un Nerval ou d'un Heine qui transmute en splendeur verbale l'échec et la mélancolie, mais un désenchantement prosaïque, qui se nourrit de détails délibérément quotidiens et platement désespérants, groupés en petites vignettes. S'il est un principe auquel Kästner demeure fidèle dans ses quatre recueils des années weimariennes, c'est bien le choix d'un certain registre, celui qui couvre linguistiquement la réalité du monde et de l'existence telle que la perçoivent à l'époque les salariés des grandes villes. Les citadins des classes moyennes, plus précisément ceux de la «lower middle class» (et encore, dans la mesure où ils ne sont ni conservateurs, ni fascistes), voilà le public auquel Kästner destine sa production poétique. Les « usagers » de sa poésie sont les cadres moyens et les employés berlinois, au premier rang desquels figurent des diplômés surqualifiés comme Jakob Fabian, l'anti-héros du célèbre roman d'actualité, obligés de se caser dans une existence étriquée ou de vivre précairement de métiers d'occasion. A ces gens, Kästner veut parler non de problèmes métaphysiques ou de grandes passions, mais de leurs problèmes quotidiens, de leurs angoisses, de leurs désillusions, de leurs rêves modestes; et il veut s'adresser à eux dans leur propre langage.

4 De là vient par exemple le charme très particulier d'un poème comme Kurzgefasster Lebenslauf, dont le titre est emprunté au langage on ne peut plus terre-à-terre des dossiers de candidature. Le poète y énumère avec la concision requise, propre à ravir 
un quelconque directeur du personnel, les étapes d'une existence qui ne se distingue en rien, extérieurement, de celle de millions de personnes : la vocation poétique récuse le nimbe de l'élection et se déguise en activité de petit travailleur indépendant : «Nun bin ich etwa 31 Jahre/Und habe eine kleine Versfabrik». La strophe finale est caractéristique : le poète ne parle plus de sa douleur sur le ton de la célébration ou de l'invocation; et l'image démonétisée de la croix que chacun doit porter durant son existence terrestre est tournée en dérision par celle du sac à dos: "Auch ich muss meinen Rucksack selber tragen! / Der Rucksack wächst. Der Rücken wird nicht breiter». La nouvelle image n'est pas simplement irrévérencieuse, elle est en outre suprêmement évocatrice: le sac à dos qu'on ne peut jamais poser, et qui s'alourdit de jour en jour alors que les forces du marcheur, dans le meilleur des cas, demeurent simplement constantes, symbolise les tares et les handicaps contingents dont l'homme est affligé, les échecs successifs dont les poids s'additionnent. L'image nous dit aussi que, dans ce monde réel où nous sommes obligés de vivre, la grâce, le salut n'existent pas, que l'individu doit porter seul le poids de sa personnalité, augmenté des expériences décourageantes par lesquelles la vie le fait passer, jusqu'à la fin du parcours. Et cela nous est dit avec le vocabulaire de la banalité quotidienne, que le talent du poète transforme en beauté parce qu'il lui donne un rythme ïambique et l'arrache ainsi à l'engluement dans la suite répétitive des jours pour l'élever au symbole.

5 La parole poétique adopte ainsi une forme d'expression et des contenus nouveaux, récusant l'emphase et le grandissement. Cette «Schnoddrigkeit» berlinoise, ce ton gouailleur que Kästner emploie constamment lorsqu'il se livre à des considérations sur le poétique, ne doit pas tromper. L'auto-dénigrement qui s'exprime dans Geständnis einiger Dichter («Lärm im Spiegel») est une coquetterie : le poète contemporain doit certes, nous dit ce poème, s'insérer dans l'existence moderne, mais non pour fournir des produits vendables, comme le lui demandent les détracteurs du lyrisme ; la poésie, au contraire, a pour mission de dénoncer le fonctionnement d'un monde entièrement soumis aux impératifs commerciaux, c'est-à-dire, comme l'affirme l'ultime strophe, de créer le "scandale ». Le "scandale ", tel que le perçoit le poète, c'est l'aliénation perpétuelle de l'homme moderne à des finalités utilitaires qui étouffent son moi profond. Et ce dernier n'est autre que le moi lyrique des âges révolus, qu'il est donc indécent désormais de célébrer ou de prétendre exprimer comme si la civilisation industrielle n'existait pas. Pourtant, ce moi enseveli n'en continue pas moins d'exister et le rôle de la poésie nouvelle est de le faire entrevoir.

6 Cette conception explique la structure du poème «Ein Baum lässt grüssen» (dans Herz auf Taillé). L'arbre qui « dit bonjour» et que le citadin («Man reist von einer Stadt zur anderen Stadt») découvre avec une bizarre stupéfaction qui lui ôte d'un seul coup sa banalité («Da sieht man draussen plötzlich eine Eiche/Es kann auch Ahorn sein. Das ist das gleiche/Denn eins steht fest: Es ist ein Baum!»), cet arbre dont il est indifférent au fond qu'il soit un chêne ou un érable, est son propre moi soudainement entr'aperçu par l'homme aliéné, à un moment de temps mort où l'ennui et la somnolence le gagnent parce qu'il ne fonctionne plus, très provisoirement, au service de finalités extérieures («... und fühlt sich frei von höheren Interessen»). La "nature» qui se dévoile ainsi, l'espace d'un instant, entre un calcul mental sur l'éventuel retard du train, la mastication d'un sandwich et une bouffée de désir sexuel pour la voisine, cette nature est en soi sans signification, et peu importe la forme qu'elle revêt. Ce qui est important, c'est que le voyageur, l'être anonyme de désir et de calcul que la civilisation moderne a engendré, dressé au service de la rentabilité du capital, recouvre la capacité de jouir de 
ce lambeau infime de nature, et du même coup redécouvre son humanité profonde, enfouie sous vingt ans de vie professionnelle (cf. les deux dernières strophes), avant de redevenir, très vite, l'être sans substance qui mange son énième sandwich au jambon en attendant de céder aux sollicitations érotiques de sa voisine.

7 L'opposition du lyrisme et du prosaïsme tourne dès lors à l'avantage du premier. Kästner n'écarte pas le prosaïsme en tonnant contre lui avec grandiloquence, il s'immerge en lui pour le subvertir.

8 Ce qui est nouveau par rapport au romantisme facile dont Kästner fustige les imitations et qui n'en finit pas d'expirer, c'est l'historicité du moi lyrique, le fait qu'il se situe nettement, délibérément, dans une époque précise, qui n'est pas évoquée uniquement par quelques détails censés faire vrai, mais par l'ensemble ou du moins par une partie considérable de sa problématique politique et sociale. Ce moi lyrique de la République de Weimar se situe aux antipodes de George et de Rilke. Il n'exerce pas un sacerdoce altier ou exclusif, il ne parcourt pas non plus, en s'isolant du monde à force d'humilité, les vastes régions d'une géographie intérieure. Il cesse d'être un moi individuel pour devenir un «nous " protéiforme. Il fréquente la rue, le bureau, le café, les lieux de vacances ou de promenade; il s'identifie à l'homme où à la femme pris comme au hasard dans la foule citadine et lui emprunte sa voix en lui prêtant son talent. Il se fond dans la masse pour montrer à l'homme des foules qu'il souffre précisément de la massification. L'autobiographie collective que le moi lyrique nous livre ainsi par fragments suscite notre intérêt non parce qu'elle est exceptionnelle, mais parce qu'elle est typique, qu'elle est à peu de détails près celle d'une génération. C'est, pourrait-on dire, un moi lyrique « unanimiste ».

Dès lors, on découvre comment l'univers poétique de Kästner s'ordonne. L'évocation de l'existence désenchantée est complétée par celle de l'existence d'avant le désenchantement: d'où la nostalgie de l'enfance ou plutôt, car il n'est plus de vert paradis, l'infantilisme. L'existence désenchantée que tout un chacun perçoit subjectivement n'est pas seulement consubstantielle à la condition humaine, elle est également le produit variable suivant les époques de conditions objectives, de déterminations générales qui fondent son historicité : d'où les thèmes de la réalité politique et sociale, de la réalité-prison, de l'antagonisme entre les "petits» et les "gros ", de l'aliénation. Cette réalité est vue dans son lieu d'élection: la grande ville, l'existence urbaine, comme dans les zones qui en forment la contre-partie illusoire: l'idylle, la nature dans la conscience du citadin, et l'érotisme. La désillusion qui frappe celui qui explore ces zones boucle le cercle: après l'avoir parcouru, on revient au désenchantement initial, mais on a appris quelque chose, on est pourvu de capacités d'analyse, sinon de capacités d'action.

10 J'examinerai successivement la thématique de l'existence désenchantée, celle de la grande ville, et le sentiment de la nature.

11 La vision de l'existence que Kästner nous transmet est cynique et morose, ce qui rend d'autant plus admirable le talent poétique qui réussit à l'exprimer de façon aussi diverse. Pour ce genre de variations, le poème-vignette, le poème-sketch (saynète) est un cadre approprié, en raison de sa brièveté même et de sa clarté, qui permettent de concentrer les effets de surprise sans risquer de lasser le lecteur.

12 La thématique de l'existence désenchantée échappe également à la monotonie du fait qu'elle est multiple. Kästner ne se contente pas de dire que la vie est dépourvue de sens (cf. «Wiegenlied» dans Herz auf Taille, notamment la strophe 5 : «Elegie ohne grosse 
Worte», dans Lärm im Spiegel, strophe 3: «Man muss sich stets die gleichen Hände waschen»; dans le même recueil : «Ein Mann verachtet sich», où les pères de famille se voient reprocher de faire des enfants qui seront des ratés à leur tour ; «Kurt Schmidt, statt einer Ballade», dans Ein Mann gibt Auskunft, préfiguration de notre « boulot, métro, dodo » d'aujourd'hui). Ce thème fondamental deviendrait très vite fastidieux si Kästner ne s'entendait pas à égayer le lecteur en inventant des manières toujours nouvelles et inattendues de truquer la banalité du langage. Ainsi dans la strophe 2 de «Ein Mann verachtet sich», où le désenchantement s'inscrit en filigrane dans les occupations peu ragoûtantes de la toilette matinale :

Ach, sein Ehrgeiz hatte falsche Zähne!

Und am liebsten spülte er sich fort

13 Ce sentiment fondamental de l'existence, ce Lebensgefühl, comme disent les Allemands à leur façon inimitable, n'est pas seulement dit et communiqué, il est analysé («Neue Sachlich-lichkeit» oblige !). L'ouvrier Kurt Schmidt, l'anti-héros de l'anti-ballade («statt einer Ballade», dit le titre, justement parce qu'il ne se passe rien, parce que le seul événement saillant est le suicide pas même héroïque du personnage dans un accès de dépression, lorsqu'il prend conscience qu'il n'échappera jamais au cycle de ses jours) n'aspire que pendant une heure sur 24 , et encore, à une existence différente, mais inconcevable, qu'il ne sait désigner que par un cliché, pauvrement évocateur à dessein : «1 Stündchen blieb für höhere Interessen» (expression qui ne désigne plus, comme tout à l'heure, dans «Ein Baum lässt grüssen», l'aliénation, mais, censément du moins, l'existence authentique). Et le père de famille anonyme dont nous entendons le monologue en style indirect libre dans Ein Mann verachtet sich, s'apparaît à lui-même dans son anonymat, son insignifiance venant précisément de ce qu'il est à la fois seul et multiple (cf. les strophes 2 et 3 ).

Ce thème essentiel de l'existence désindividualisée, décolorée, Kästner se garde toutefois de le ressasser. Il est là simplement pour relier entre elles les vignettes très diverses qui constituent toute une phénoménologie burlesque du dégoût de vivre.

Relisons le poème intitulé «Kleine Führung durch die Jugend», dans Herz auf Taille. Le Berlinois éprouve une nausée qu'il croit devoir au déracinement, alors qu'elle vient en réalité du découragement, du vieillissement prématuré que cause une vie terne et sans issue. Pour retrouver ce que l'on appelle aujourd'hui ses racines, il décide de se rendre dans la petite ville de province où il a grandi. Mais ce retour aux origines, loin de lui redonner force et vigueur, redouble son accablement : il redécouvre la stagnation de ses années de jeunesse («Es sind Stationen der Vergangenheit/Man dachte, sie sei tot. Sie blieb hier wohnen.») ; et l'école, lieu privilégié de l'enfance qu'il voulait revoir, lui apparaît dans le dépouillement fantastique de l'existence nue ;

Dann steigt man auss. Und zögert. Und erschrickt.

Der Wind steht still, und alle Wolken warten.

Man biegt um eine Ecke. Und erblickt

ein schwarzes Haus in einem kahlen Garten.

16 Après les battements de cœur soulignés par les césures des premiers vers, après l'effarement paralysant qu'expriment l'image et les allitérations du deuxième, le sentiment d'atonie qui prédomine est communiqué par le rythme qui, au troisième et au quatrième vers, se défait pour se rapprocher de l'allure de la prose.

17 Le passé personnel oppressant n'apporte plus rien, lorsqu'on le ressuscite, à celui qui doit subir une existence désolée. Le moi lyrique, ayant manqué son retour à l'enfance, 
n'a plus comme domaine réservé que l'infantilisme. Kästner, auteur de nombreux livres bien connus pour enfants, n'use que parcimonieusement de cette ressource dans ses recueils poétiques. Citons toutefois: «Verhinderte Weihnachten, Pädagogik spasseshalber, Hochzeitmachen, Die Existenz im Wiederholungsfalle», dans Lärm im Spiegel; et «Genesis der Niedertracht» dans Ein Mann gibt Auskunft.

Le présent ? C'est le quotidien qui vous contraint de toutes parts et qu'il est impossible de transformer. Comme Sartre quelques années plus tard (dont il préfigure les écœurements et le penchant pour un comique nihiliste), Kästner ressent la stagnation du présent à travers la fixité des choses, qui sont là, immuables, dans leur superfluité. C'est ce qu'exprime le poème «Trottoircafés bei Nacht», dans Herz auf Taille:

An den Garderobenständen

schaukeln Hüte, und der Abendwind

möchte sie in Obst verändern,

Aber Hüte bleiben, was sie sind.

Comme au fond rien ne bouge, le temps historique piétine et les événements d'une vie deviennent impersonnels, comme devient informe et floue la personnalité du sujet auquel ils arrivent (cf. «Kurzgefaster Lebenslauf», strophes 3 et 4). Les biographies et les personnes paraissent interchangeables, le défunt sujet autonome des philosophies idéalistes flotte dans le vide. Et lorsqu'il n'est pas un intellectuel, lorsqu'il n'est pas capable de s'élever au-dessus des choses par l'ironie, il ne lui reste plus que des expériences totalement frustrantes: l'amour impossible, la sexualité sans amour, le mariage-prison, la révolte impuissante (cf. «Das Gemurmel eines Kellners», dans Lärm im Spiegel), l'évasion ridiculement manquée (voir l'histoire du provincial qui se retrouve complètement à sec, plumé, dans une chambre d'hôtel à Berlin et adresse une lettre embarrassée à son épouse pour qu'elle lui envoie des vêtements et un peu d'argent, lettre versifiée sous l'intitulé «Brief eines nackten Mannes» dans Lärm im Spiegel).

Quant à la mort, elle est désacralisée, intégrée au quotidien. Telle nous la présente le poème «Gedanken beim Überfahrenwerden» dans Ein Mann gibt Auskunft: nous assistons à l'interruption fortuite d'une existence quelconque, éprouvée comme une contrariété.

21 L'univers urbain chez Kästner est à l'image de l'existence désenchantée. Il est le lieu de l'anonymat, du travail aliénant et répétitif, de la débauche monotone, des amourettes et des liaisons sans lendemain, qui se dissolvent dans la lassitude (cf. «In der Seitenstrasse», dans Ein Mann gibt Auskunft). De Berlin, nous ne voyons ni les monuments, ni les quartiers élégants, mais seulement des immensités toutes pareilles, interchangeables, comme les individus qui s'y entassent. Ainsi dans «Nächtliches Rezept für Städer» («Ein Mann gibt Auskunft») :

Es gibt so viele Strassen, ach so vièle!

Und hinter jeder Biegung sind es mehr.

Berlin est-il pour autant uniquement une machine à déraciner et à user les individus ? Certes, Kästner ne nous en montre aucune qualité positive. Mais la ville de province natale, avec ses souvenirs ternes et pesants, est, nous l'avons vu dans Kleine Führung durch die Jugend, pire encore à tout prendre :

Man geht, denkt an die kleinen Eisenbetten

und fährt am besten wieder nach Berlin.

23 A l'inverse, les gens de la campagne (cf. «Besuch von Lande», dans Ein Mann gibt Auskunft) ne trouvent à Berlin qu'un bruit infernal et incessant, une prostitution 
voyante, des lumières criardes, une circulation monstrueuse, et cette expérience les plonge dans un état cataleptique.

Une ville inhospitalière donc, où la quête personnelle n'a pas de sens. Tel est l'enseignement que nous communique Nächtliches Rezept für Städter, qui peut être lu comme une caricature nihiliste du parcours initiatique. Le poète prescrit de prendre un autobus pour n'importe quelle destination, puis de marcher au hasard. Après avoir ainsi déambulé, on parvient à la révélation de son propre vide intérieur et de son impuissance face à la bêtise de la masse.

Man nehme sich bei dem Spaziergang Zeit.

Er dient gewissermassen höhern Zwecken.

Er soll das, was vergessen wurde, wecken.

Nach zirka einer Stunde ist's soweit.

Nun weiss man wieder, was man wissen muss,

statt dass man in Zufriedenheit erblindet:

dass man sich in der Minderheit befindet!

Chez Kästner, la grande ville n'est pas, contrairement par exemple au Paris de Balzac, un monde qu'il faut conquérir, où les réussites les plus éclatantes sont rendues possibles sur un fond de tares sociales. Elle est la matérialisation d'un message indéfiniment répété, une immensité dont le paradoxe est qu'elle génère l'enfermement. Il est donc vain de chercher dans ces vignettes un documentaire sur les réalités citadines objectives, sur le Berlin grouillant de l'époque que décrivent entre autres les romans de Hans Fallada et de Christopher Isherwood. Le poète ne sort pas de lui-même, il annexe la grande ville à son décor intérieur, en fait le signe de sa solitude et de sa vacuité.

Avec Kästner, nous sommes loin de l'imagerie expressionniste, de sa stratégie de la projection et du grandissement. Le monde urbain n'est plus chez Kästner un espace mythique: il ne dévoile pas l'emprise du mal, il se borne à faire apparaître le dysfonctionnement d'une machine sociale dont la logique produit à jet continu des existences manquées. Là où l'expressionnisme nimbait la cité moderne d'une horrible grandeur, Kästner ne découvre que le dérisoire, la mesquinerie, l'accablement naissant de l'uniformité, du recommencement perpétuel et inutile d'existences médiocres, qui sont à peu près toutes semblables.

Dans la grisaille citadine, la nature est objet de désir, elle représente dans la conscience de l'habitant des métropoles le lieu du bonheur possible, l'endroit où l'on peut se soustraire aux mécanismes socio-économiques et à la routine désespérément laborieuse qu'ils engendrent. Il s'agit d'une nature vue par le citadin et qui apparait par conséquent sous deux aspects : l'exotisme et l'idylle. D'une part le mirage des grands espaces, d'une vie libre et gratifiante; de l'autre, les lieux de détente apparemment accessibles : la plage, la montagne à ski, les parcs. Or, dans les deux cas, c'est une nature apprivoisée, mise aux mesures du «kleiner Mann», du petit-bourgeois en quête d'attendrissements, d'émerveillements, d'évasion.

L'exotisme naïf inspire les premières strophes gentiment ironiques de «In der Seitenstrasse» (Ein Mann gibt Auskunft) : aspiration à s'évader vers des îles lointaines qui seraient telles que les montrent les romans et les gravures, et où il serait possible d'échapper à l'envahissante foule berlinoise : «Dort haben es Liebespaare besser!». Dans "Trottoircafés bei Nacht», cet exotisme est réduit à sa plus simple expression: sept palmiers en pot, que le patron a achetés en solde... (cf. Herz auf Taille). 
29 Le poète «neusachlich» ne s'appesantit pas sur cette nostalgie factice, avatar d'un romantisme de pacotille. Par contre, il évoque plus longuement, et même avec beaucoup de sympathie, l'attendrissement du citadin à la vue du printemps qui s'installe. C'est le sujet du poème «Besagter Lenz ist da» (dans Herz auf Taille).

Comme il fallait s'y attendre, le titre du poème n'est pas innocent. Kästner raille le sérieux grotesque du jargon administratif. L'ironie est également présente dans les notations prosaïques à dessein de la première strophe ;

Es ist schon so. Der Frühling kommt in Gang.

Die Luft ist weich, als wäre sie aus Daunen.

Mais le moi poétique manifeste son pouvoir de transfiguration dans le vers intermédiaire ;

Die Bäume räkeln sich. Die Fenster staunen.

Ironie encore, mais douce, sans agressivité, lorsque l'émoi du citadin s'exprime avec une talentueuse pseudo-naïveté dans le vocabulaire de la civilisation urbaine : «Am Himmel tanzen blanke Aéroplane», «Die Welt wird frisch gestrichen»: décor des chambres d'enfant aux couleurs rutilantes. Toujours est-il que la venue du printemps, malgré la pondération du sceptique revenu de tout, n'est pas réductible à une récurrence mécanique, qu'elle a gardé son pouvoir d'émerveillement ;

Es ist zwar jedes Jahr dieselbe Sache,

doch ist es immer wie zum erstenmal.

C'est bien, en effet, un phénomène primordial, archétypal, qui crée un rapport entre les besoins psychiques de l'homme et un cosmos qui - une fois n'est pas coutume - semble proche, plein de sollicitude, humanisé en un mot. Rapprochement qu'atteste le nombre relativement élevé de métaphores, un procédé qui revêt chez Kästner un caractère tout à fait exceptionnel.

Même sensation d'éveil dans «Prima Wetter» (Ein Mann gibt Auskunft). Le petitbourgeois y aspire même à l'envol, presque à l'existence esthétique. Mais le ton est déjà un peu grinçant. Le printemps, dans ce poème, est humanisé de façon parfois caricaturale («Auf weissen Wolken wie auf Sofas liegen») et sa célébration dévalorisante se termine sur une interrogation nihiliste ;

Es ist, um förmlich aus der Haut zu fahren.

Die grosse Schwierigkeit ist nur: Wohin?

Souvent, la nature idyllique est gâchée - par la massification envahissante qui déborde des cités. C'est le thème de «Misanthropologie» (Ein Mann gibt Auskunft), où le charme conventionnel d'un endroit agreste est saccagé par une horde de promeneurs; un thème similaire est développé dans «Selbstmord im Familienbad» (ibid.). L'homme des foules est toujours ramené à sa condition : ici encore, on retrouve le thème lancinant de l'évasion manquée ou impossible. Le vocabulaire délibérément banal marque le refus de la poétisation factice; en même temps, la subversion ironique du langage quotidien employé par l'homme aliéné souligne la volonté de rejeter le prosaïsme - comme le kitsch, qui n'en est au fond qu'une tentative de compensation maladroite. D'où la prédilection pour les formulations où le sentiment de la nature s'exprime dans le langage de la civilisation urbaine.

On a souvent critiqué, à juste titre sans doute, l'inefficacité politique du lyrisme d'Erich Kästner ${ }^{4}$. Il est d'autant plus curieux qu'au bout d'un demi-siècle où l'Histoire avec majuscule a surabondamment déployé ses paradoxes et ses horreurs, ce lyrisme, qui 
pourtant était soucieux au premier chef de s'inscrire dans son époque, n'ait rien perdu de son actualité.

Cette actualité est attestée par les rééditions en livre de poche. La poésie de Kästner a survécu parce qu'elle est authentiquement populaire, parce qu'elle exprime la condition des foules telle qu'elle est encore aujourd'hui - psychologiquement, sinon pécuniairement. Le lecteur y retrouve à la fin du XXe siècle, par-delà les bouleversements et les convulsions de la deuxième guerre mondiale, de la guerre froide et de la décolonisation, son expérience quotidienne, a savoir l'engluement dans l'existence infra-historique : recherche des petites joies, aspirations modestes, face au grand désenchantement, à l'oppression de l'anonymat, à la sensation d'être l'objet insignifiant des grandes forces qui mènent le monde, d'avoir un destin interchangeable avec celui du voisin.

Populaire au meilleur sens du terme, c'est-à-dire refusant l'amplification des fantasmes collectifs et la démagogie, ce lyrisme l'est également par sa mélodie propre et ses «Bauformen» qui sont au service d'une tonalité où se mêlent la sensibilité vraie (celle qui se met à l'écoute des problèmes de l'homme contemporain) et une ironie typiquement berlinoise, ennemie des grands mots, des grands sentiments et de toute affectation.

\section{NOTES}

1. - Sur le lyrisme de Kästner, on peut consulter :

Volker Klotz, «Notizen zu neusachlichen Gedichten Erich Kästners», dans Historizität in Sprachund Literaturwissenschaft, Munich, 1974, p. 479 sq.

Dirk Walter, Zeit kritik und Idyllensehnsucht. Erich Kästners Frühwerk (1928-1933) als Beispiel linksbürgerlicher Literatur in der Weimarer Republik, DISS., Cologne, 1976.

Egon Schwarz, «Die strampelnde Seele. Erich Kästner in seiner Zeit», dans Dichtung, Kritik, Geschichte. Essays zur Literatur 1900-1930, Göttingen, 1983.

Helmut Kiesel, Erich Kästner, Munich, 1981.

2. - Ces quatre recueils ont été réédités au Deutscher Taschenbuch Verlag (dtv) en 1989.

3. - Ernst Glaeser, «Erik Reger. Zu seinem Roman "Union der festen Hand"», dans Die literarische Welt 7 (21 août 1931). Texte réimprimé dans : Anton Kaes (éd.), Weimarer Republik. Manifeste und Dokumente zur deutschen Literatur 1918-1933, J.B. Metzlersche Verlagsbuchhandlung, Stuttgart, 1983. Le roman Union der festen Hand a été redite au Scriptor Verlag, Königstein/Taunus, en 1978.

4. - Dès 1931, Walter Benjamin se voyait refuser par la Frankfurter Zeitung un compte rendu très défavorable du recueil Ein Mann gibt Auskunft («Linke Melancholie. Zu Erich Kästners neuem Gedichtbuch»). Le texte en est reproduit dans Walter Benjamin, Schriften III, Suhrkamp, Frankfurt-am-Main, 1972. 


\section{RÉSUMÉS}

Le lyrisme de Kästner est populaire aujourd'hui encore, malgré son message provocant de désillusion, qui lui a valu de nombreuses réactions de rejet. Dans cet article, on n'étudie pas l'attitude politique du poète, mais son mode personnel d'expression de soi, ce qui chez lui est littérature, lyrisme à proprement parler, par opposition à la froideur «néo-objectiviste » qu'il arbore et à sa gouaille typiquement berlinoise. Kästner rompt de manière ostentatoire avec le romantisme prolongé, avec la conception traditionnelle du moi lyrique; il renonce à la confession, à l'anthropomorphisation de la nature, au rapport à la transcendance, au style sublime, pour devenir l'expression polyphonique de l'âme citadine. Le sentiment d'aliénation, la conscience d'être un médiocre, un n'importe qui, un être interchangeable, la banalité des phantasmes d'évasion qui caractérisent l'individu salarié dans l'univers du rendement, son langage jargonnant trahissant par ses clichés mêmes un accablant mutisme, voilà la matière des quatre volumes de poèmes publiés par Kästner durant les années de la République de Weimar. Le talent du poète est d'avoir triomphé de ce prosaïsme omniprésent et sans issue par le pouvoir d'émotion d'une tonalité combinant l'esprit de dérision parfois nihiliste et une tendresse imprégnée d'humanisme.

Kästners Lyrik der Weimarer Zeit ist bis heute volkstümlich geblieben, obwohl sie provokativ als desillusionierende Dichtung auftritt und daher oftmals verfemt wurde. Untersucht wird in diesem Aufsatz nicht die politische Einstellung des Dichters, sondern seine besondere Art der intimen Selbstaussprache, m.a. W. was an ihm Literatur ist, das eigentlich Lyrische bei aller Zurschaustellung neusachlicher Kühle und Berliner Schnoddrigkeit. Kästner kehrt der romantisierenden Dichtung und dem tradierten lyrischen Ich ostentativ den Rücken, verzichtet auf Selbstbekenntnisse, Naturbeseelung, Transzendenzbezug und hohen Stil und wird zum polyphonen Ausdruck der grosstädtischen Massenseele. Die Selbstentfremdung, die Mittelmässigkeit, Beliebigkeit und Austauschbarkeit, die banalen eskapistischen Phantasien des lohnabhängigen Individuums in der Leistungswelt, seine Sprachnot, wie sie sich im klischierten Jargon kundgibt, bilden den Stoff der vier Gedichtsammlungen, die Kästner in der Zeit der Weimarer Republik herausgab. Das Talent des Dichters liegt in der Überwindung dieser scheinbar auswegslosen, allgegenwärtigen Prosaizität durch das Erschütterungsvermögen einer Tonart, die den manchmal nihilistisch anmutenden Witz mit einer tiefen humanistischen Zärtlichkeit vereinigt.

\section{AUTEUR \\ PIERRE VAYDAT}

Université Charles-de-Gaulle - Lille III 